\title{
Rousseaus philosophischer Anarchismus. Der demokratische Gesellschaftsvertrag und das Problem der politischen Verpflichtung ${ }^{1}$
}

\author{
Robin Celikates
}

\section{Rousseau's Philosophical Anarchism: The Democratic Social Contract and the Problem of Political Obligation}

\begin{abstract}
In this article I firstly sketch the current discussion of the problem of political obligation and the position of philosophical anarchism. In the second step, Rousseau's answer to this problem is interpreted as a variant of aposteriori philosophical anarchism. Thirdly, I show under which conditions, according to Rousseau, the political obligations of citizens cease to exist or become qualified, and finally I raise the question whether a specifically Rousseauian perspective on civil disobedience can be developed against this background.
\end{abstract}

Keywords: Rousseau, Political Obligation, Anarchism, Contract Theory, Democracy

Schlagwörter: Rousseau, politische Verpflichtung, Anarchismus, Vertragstheorie, Demokratie

\section{Einleitung}

Für die einen ist er „one of the most sinister and most formidable enemies of liberty in the whole history of modern thought" (Berlin 2002, S. 49), für die anderen Verfechter demokratischer Freiheit, „the theorist par excellence of participation" (Pateman 1970, S. 22). Wie diese wohlvertraute Bandbreite der Interpretationen bezeugt, ist die Frage, wie Rousseaus Politische Theorie sich - über die ideengeschichtliche Einordnung hinaus - auf die gegenwärtigen Herausforderungen der Demokratietheorie beziehen lässt, notorisch schwierig zu beantworten. Zuletzt wurden jedoch nicht nur theoretische, sondern auch direkter praktische Aktualisierungsversuche unternommen. So hat Benjamin Barber (2012) jüngst auf der Aktualität von Rousseaus Analyse und Kritik der wechselseitigen Verstärkung ökonomischer und politischer Ungleichheiten sowie des hinter der Fassade repräsentativer Parteiendemokratien stattfindenden Abbaus demokratischer Selbstbestimmung insistiert und Rousseau in die Reihen von Occupy Wall Street aufzunehmen versucht.

Die folgenden Überlegungen zur demokratietheoretischen Aktualität Rousseaus setzen sehr viel abstrakter ein, nämlich mit einer Exposition des Problems der

1 Für hilfreiche Kommentare danke ich den Teilnehmerinnen und Teilnehmern der Konferenz „Ambivalenzen demokratischer Freiheit“ in Frankfurt a. M. (Juni 2012), der „2a Semana de filosofía práctica“ in Ujué (August 2012) und des Workshops „Walking With Rousseau“ in Genf (September 2012), zwei anonymen Gutachtern der PVS sowie Michael Bloch, Felix Koch, Fred Neuhouser und Michaela Rehm. 
politischen Verpflichtung - also der Frage, ob es eine Pflicht gibt, den Gesetzen zu gehorchen, weil es die Gesetze sind -, wie es in der gegenwärtigen Politischen Philosophie diskutiert wird. In einem zweiten Schritt werde ich Rousseaus Antwort auf dieses Problem skizzieren, das meines Erachtens im Zentrum seiner Politischen Theorie steht, und diskutieren, wie diese sich in die gegenwärtigen Debatten einordnen läßt. Drittens werde ich fragen, unter welchen Bedingungen die politische Verpflichtung der Bürger Rousseau zufolge erlischt oder zumindest nur noch eingeschränkt gilt. Abschließend werde ich viertens doch wieder auf Barbers Aktualisierungsversuch zurückkommen, allerdings in etwas anderer Absicht, indem ich die Frage aufwerfe, ob es eine spezifisch rousseauistische Perspektive auf zivilen Ungehorsam gibt - eine Form politischen Handelns, die sich in der Praxis wieder einiger Beliebtheit erfreut, deren demokratietheorischer Status allerdings weiterhin umstritten ist.

\section{Das Problem der politischen Verpflichtung}

Warum halten wir uns an die Gesetze? In den meisten Fällen werden wir eine Reihe unterschiedlicher Motive haben, von Angst vor Bestrafung über bloße Gewohnheit zu moralischer Überzeugung und Loyalität gegenüber der Rechtsordnung. Rousseau hat an dieser empirischen Frage ein sehr viel größeres - sowohl kritisches als auch konstruktives - Interesse als die gegenwärtige Politische Philosophie, die sich von der Perspektive der Politischen Soziologie weitgehend entkoppelt hat, zu deren ersten Vertretern Rousseau gezählt werden kann. ${ }^{2}$

Ihre eigentlich philosophische Sprengkraft entfaltet die Frage nach dem Gesetzesgehorsam aber erst, wenn sie nicht als empirische, sondern als normative Frage formuliert wird: Warum sollten wir uns an die Gesetze halten? Und diese Frage steht eindeutig im Zentrum von Rousseaus Gesellschaftsvertrag, der aus diesem Grund den Untertitel „Prinzipien des Staatsrechts“ (principes $d u$ droit politique) trägt. ${ }^{3}$

Das Problem, das durch diese Frage aufgeworfen wird und das die Politische Philosophie seit ihren Anfängen bei Platon umtreibt, wird heute meist als das Problem der politischen Verpflichtung (political obligation) bzw. der politischen Autorität bezeichnet (vgl. Rinderle 2005; Celikates 2011). Dabei geht es nicht um die de facto-Macht, jemanden zum Rechtsgehorsam zwingen zu können, sondern um die de jure-Autorität, die mit dem Recht verbunden ist, bindendes Recht setzen, anwenden und durchsetzen zu dürfen (also, im weiteren Sinn, zu regieren - aus diesem Grund wird auch von einem „right to rule“ gesprochen). Diese Autorität

2 Dies zeigen vor allem die institutionentheoretischen Teile des Gesellschaftsvertrags in den Büchern III und IV, vor allem aber Rousseaus Schriften zu Korsika und Polen (Rousseau 1964, S. 901 ff., 953 ff.; im Folgenden wird auf diese Ausgabe der Euvres complètes verwiesen als OC III + Seitenzahl); vgl. dazu insgesamt die nicht mehr viel gelesenen, aber sehr lesenswerten Kommentare zum Gesellschaftsvertrag von Emile Durkheim (2008) und Maurice Halbwachs (1943).

3 So betont Rousseau neben der Wichtigkeit empirischer, stets kontextabhängiger Realisierungsbedingungen immer auch die normative - de jure - Perspektive und fragt nach den „Grundsätzen, die für alle gelten“ (Rousseau 2013, II.11, S. 67 [OC III, S. 393]); vgl. auch Rousseau 2013, IV.8, S. 163 (OC III, S. 467): „Aber lassen wir diese politischen Betrachtungen beiseite, kehren wir zum Recht zurück und legen wir in dieser wichtigen Angelegenheit die Prinzipien fest." 
geht, zumindest einer weit verbreiteten Annahme zufolge, wesentlich mit einem Recht auf Gehorsam einher sowie mit der entsprechenden Pflicht der Adressaten zum Rechtsgehorsam, also dazu, sich an die Gesetze zu halten, weil es die Gesetze sind. Bei dieser Pflicht handelt es sich freilich nicht um eine rechtliche Pflicht (denn es versteht sich von selbst bzw. ist tautologisch, daß es eine rechtliche Pflicht gibt, sich an die Gesetze zu halten), sondern um eine moralische Pflicht.

Die entscheidende Frage ist dann, ob es eine derartige Pflicht gibt. Auf Gefühle des Verpflichtetseins und andere de facto-Einstellungen zu verweisen, stellt keine ausreichende Antwort dar, denn es lässt sich immer fragen, ob diese Gefühle und Einstellungen angemessen sind, und dabei muss man, wie gerade Rousseau betont (etwa in der Analyse des betrügerischen Gesellschaftsvertrags im Zweiten Diskurs), stets die Möglichkeit von Formen falschen Bewusstseins, also eines systematisch verzerrten Legitimitätsglaubens, in Rechnung stellen. Nimmt man die Frage als normative Frage ernst, so lassen sich, grob vereinfachend, drei mögliche Antworten identifizieren, die sich in der Geschichte der Politischen Philosophie unterschiedlich großer Beliebtheit erfreut haben: (1) Ja, es gibt eine solche Pflicht und sie ist unbedingt bzw. absolut (so etwa derart unterschiedliche Autoren wie Platon, Hobbes und Kant, manchen zufolge auch Rousseau, bei dem man bekanntlich vergeblich nach einem Widerstandsrecht sucht). (2) Ja, es gibt eine solche Pflicht, aber sie ist durch die Genese und den Inhalt des Rechts bedingt - unterliegt also prozeduralen und substantiellen Einschränkungen - und gilt daher prima facie bzw. pro tanto, auch wenn sie im Normalfall vorrangig ist (so etwa Locke und zahlreiche Vertreter der Tradition des Naturrechts, manchen zufolge auch Rousseau). (3) Nein, es gibt keine derartige Pflicht, zumindest nicht im Normalfall (so die Vertreter des politischen und philosophischen Anarchismus - und, so möchte ich zeigen, zumindest in manchen Hinsichten auch Rousseau).

Viele der gängigen Argumente für die erste Position finden sich bereits in Platons Dialog Kriton. Der auf die Vollstreckung des Todesurteils wartende Sokrates lehnt es dort ab, aus dem Gefängnis zu fliehen, und erläutert, warum er auch angesichts offensichtlicher Ungerechtigkeit zum Rechtsgehorsam verpflichtet ist. Als Grund dieser Verpflichtung gibt er an, wozu die Gerechtigkeit ihn verpflichte (auf keinen Fall Unrecht zu tun), wer er sei (ein Bürger Athens, der, wie ein Sohn im Verhältnis zu seinem Vater, aufgrund seiner Identität und Rolle zum Gehorsam verpflichtet ist), was er erhalten habe (die vielfältigen Vorteile des Lebens in der Polis, die ihn aus Dankbarkeit und Fairness zu Gehorsam verpflichten) und was er getan habe (nämlich am politischen Leben teilgenommen und damit seine $\mathrm{Zu}$ stimmung zum Ausdruck gebracht sowie auf Auswanderung verzichtet) (vgl. Preuss 1984, 36-47). In der gegenwärtigen Diskussion lassen sich daran anschließend, wieder grob vereinfachend, folgende drei Familien von Ansätzen zur Begründung von politischer Verpflichtung (neben pluralistischen Kombinationsversuchen) unterscheiden, wobei die entsprechenden Pflichten heute meist nicht mehr als absolut, sondern als bedingt verstanden werden:

(1) Naturrechtlichen Ansätzen zufolge ergibt sich die Gehorsamspflicht aus natürlichen Pflichten etwa zur Förderung oder Ermöglichung von Gerechtigkeit oder zur Sicherung gleicher Freiheit (so etwa Kant, in manchen Lesarten aber auch Rousseau; vgl. Stilz 2009). Wie vor allem Simmons (2008) argumentiert, sind diese 
Ansätze, denen zufolge politische Verpflichtungen allgemeine, nicht freiwillig übernommene Verpflichtungen sind, mit dem sogenannten Problem der Partikularität konfrontiert. Die zugrundeliegende Pflicht ist nämlich allgemeiner Natur und bezieht sich beispielsweise auf die Förderung von Gerechtigkeit (bzw. gerechter Institutionen) an sich. Es bleibt daher unklar, wie wir als Bürgerinnen und Bürger bestimmter Staaten auf dieser Basis zum Gesetzesgehorsam verpflichtet sein können: Warum müssen wir die gerechten Institutionen unseres eigenen Staates und können nicht die eines anderen, vielleicht sogar gerechteren Staates unterstützen oder auf die Einrichtung einer globalen gerechten Ordnung hinarbeiten?

(2) Assoziativen Ansätzen zufolge ergibt sich die Gehorsamspflicht aus sozialen Rollen und Identitäten (so etwa Vertreter des Kommunitarismus, aber auch Ronald Dworkin sowie in manchen Lesarten Rousseau; vgl. etwa Horton 2010). Bei politischen Verpflichtungen handelt es sich dieser Position zufolge um besondere, nicht freiwillig übernommene Verpflichtungen, die in den spezifischen Beziehungen der Bürgerinnen und Bürger zueinander begründet sind und daher nicht mit dem Partikularitätsproblem konfrontiert zu sein scheinen. Um die unplausible Annahme zu vermeiden, dass jede Person, die sich in der Rechtsposition einer Bürgerin befindet, ipso facto einer Gehorsamsplicht unterliegt, wird diese Pflicht meist auf legitime Staaten eingeschränkt und in legitimen Beziehungen wechselseitiger Erwartungen und Festlegungen begründet. Aus normativer Perspektive stellt sich dann jedoch die Frage, inwiefern faktische Rollen, Identitäten oder Beziehungen tatsächlich auf eigenständige Weise Verpflichtungen der benötigten Stärke generieren können und ob die Existenz derartiger Verpflichtungen gegebenenfalls nicht auf andere, von den infragestehenden Rollen, Identitäten oder Beziehungen unabhängige normative Erwägungen (etwa in Form von Legitimitätskriterien) zurückzuführen ist. Dies können wir als das Problem der unabhängigen normativen Relevanz bezeichnen.

(3) Transaktionsbasierten Ansätzen zufolge ergibt sich die Gehorsamspflicht aus empfangenen Vorteilen oder aus expliziter oder impliziter Zustimmung. $\mathrm{Zu}$ dieser Theoriefamilie gehören demnach zwei Arten von Positionen: (a) solche, denen zufolge politische Verpflichtungen besondere, nicht freiwillig übernommene Verpflichtungen darstellen, die sich aus normativ signifikanten Transaktionen und den daraus empfangenen Vorteilen ergeben und entweder als Pflichten der Fairness oder der Dankbarkeit zu verstehen sind; (b) solche, denen zufolge politische Verpflichtungen besondere, freiwillig übernommene Verpflichtungen darstellen, die auf Handlungen unsererseits - nämlich einen freiwilligen Akt der Verpflichtung - zurückzuführen sind. Die erste Variante ist vor allem mit dem Problem der Freiwilligkeit konfrontiert, da unklar bleibt, zu welcher Gegenleistung wir aus welchen Gründen verpflichtet sind, wenn wir die fraglichen Vorteile gar nicht freiwillig empfangen haben bzw., wie im Fall vieler öffentlicher Güter, gar nicht in der Lage sind, sie abzulehnen. Auf den Einwand gegen die zweite Variante, dass eine tatsächliche explizite Zustimmung nur unter seltenen Ausnahmebedingungen angenommen werden kann, wird von denen, die die Existenz politischer Verpflichtung verteidigen möchten, unter anderem mit einer Ermäßigung des Zustimmungskriteriums reagiert, indem dieses im Sinne impliziter, dispositionaler oder hypothetischer Zustimmung reformuliert wird. Diese Aufweichung des Zustim- 
mungskriterium scheint jedoch die normative Überzeugungskraft und/oder Eigenständigkeit dieser Variante des transaktionsbasierten Modells zu unterminieren, so dass letztlich, folgt man Simmons (2008), doch nur das Kriterium der tatsächlichen Zustimmung (ob nun explizit oder implizit) als plausibler Kandidat übrigbleibt - allerdings ist dieses Kriterium, wie gesehen, äußerst anspruchsvoll und in den Augen vieler Theoretiker unrealistisch, da die Bürgerinnen und Bürger real existierender Demokratien von wenigen Ausnahmen abgesehen nicht in dem geforderten Sinn ihre Zustimmung erteilt haben oder auch nur erteilen könnten (Problem der overdemandingness). Handelt es sich jedoch tatsächlich um das einzig stichhaltige Kriterium, so scheint daraus zu folgen, dass nur sehr wenige Bürgerinnen und Bürger einer genuinen Verpflichtung unterliegen und dass nur sehr wenige Staaten die Autorität besitzen, die sie für sich in Anspruch nehmen. Genau diese Schlussfolgerung wird von Vertretern des Anarchismus aus der skizzierten Diskussion gezogen (Simmons 2001).

Hinter dem Label „Anarchismus“ verbergen sich jedoch unterschiedliche, und vor allem: unterschiedlich starke Positionen. Zunächst muss man den apriorischen vom aposteriorischen Anarchismus unterscheiden (vgl. etwa Rinderle 2005, S. 22-23). Während für ersteren aus prinzipiellen bzw. begrifflichen Gründen ausgeschlossen ist, dass es so etwas wie legitime Herrschaft bzw. eine kohärente Begründung staatlicher Autorität geben kann (so etwa Wolff 1998), beschränkt sich letzterer auf die Behauptung, alle existierenden und zumindest die meisten historisch bekannten Formen staatlicher Herrschaft seien illegitim und ihre Autoritätsansprüche daher nicht überzeugend begründbar. Der aposteriorische Anarchismus wird etwa von Simmons (2001) vertreten, dem zufolge die tatsächliche Zustimmung der Herrschaftsunterworfenen den Staat tatsächlich legitimieren würde, bisher in der Praxis aber nicht als eingelöst und unter den existierenden Bedingungen noch nicht einmal als einlösbar unterstellt werden kann. Demnach ist es zwar nicht unmöglich, unter den gegebenen Bedingungen jedoch äußert unwahrscheinlich, dass ein Staat die genannten Legitimitätsanforderungen erfüllt und dass seine Subjekte daher die entsprechenden politischen Verpflichtungen haben. Diese schwächere und überzeugendere Variante des Anarchismus geht davon aus, dass aus der Tatsache, dass kein gegenwärtiger Staat diese Bedingungen erfüllt, nicht folgt, dass kein Staat diese Bedingungen erfüllen kann. Aus diesem Grund handelt es sich um einen aposteriorischen oder, wie man auch sagen könnte, einen sekundären Anarchismus: Die anarchistische Konsequenz wird nicht vorausgesetzt, sondern folgt aus der Anwendung eines nicht selbst anarchistischen Legitimitätskriteriums, das sich aus dem zweiten der oben diskutierten transaktionsbasierten Ansätze ergibt (sich im Prinzip aber natürlich auch aus der Anwendung der anderen Kriterien ergeben kann). ${ }^{4}$

4 Entsprechend könnte man mit Blick auf meine erste Unterscheidung auch sagen, dass die Autoren, die von einer bedingten Gehorsamspflicht ausgehen, letztlich dieselbe Position wie die philosophischen Anarchisten vertreten - das würde jedoch eine wichtige Differenz verdecken: Während die Vertreter der oben (S. 670) unter (2) genannten Position für den Normalfall eine Gehorsamspflicht annehmen, die im Ausnahmefall außer kraft gesetzt werden kann, sehen es die Vertreter des philosophischen Anarchismus (3) gerade als den Normalfall an, dass eine solche Pflicht nicht vorliegt, die in Ausnahmefällen aber durchaus vorliegen könnte. 
Ebenso wichtig wie die Unterscheidung zwischen apriorischem und aposteriorischem Anarchismus ist die zwischen politischem und philosophischem Anarchismus. Der philosophische Anarchismus ist insofern negativ, als es ihm zufolge keinen moralischen Grund gibt, der als solcher zum Gehorsam gegenüber dem Staat verpflichtet. Es gibt also keine moralische Pflicht zum Rechtsgehorsam bzw. keine genuine politische Verpflichtung. Anders als die Vertreter des politischen Anarchismus annehmen, folgt hieraus dem philosophischen Anarchismus zufolge aber umgekehrt keineswegs schon die moralische Pflicht, sich gegen den Staat zu wenden, vielleicht nicht einmal das Recht dazu. Ob es ein Recht oder gar eine Pflicht gibt, sich den Gesetzen zu widersetzen, muss aus dieser Sicht erst in einem weiteren Argumentationsschritt geklärt werden. Dass ein Gesetz ein Gesetz ist, ist zwar an sich kein Grund, sich an es zu halten, aber in den meisten Fällen wird es dieser Position zufolge andere, etwa prudentielle oder moralische Gründe geben, sich an bestimmte Gesetze wie zum Beispiel die Straßenverkehrsordnung oder das Strafgesetzbuch zu halten. Anarchistisch ist diese Position also primär im philosophisch grundlegenden Sinn, dass sie die Unterstellung politischer Verpflichtung zurückweist - die praktisch-politischen Implikationen dieser Zurückweisung stehen auf einem anderen Blatt.

Folgt man Simmons, so spricht aufgrund der Schwierigkeiten, in die die oben skizzierten Positionen geraten, einiges für die Kombination der Zustimmungstheorie politischer Verpflichtung mit dem aposteriorischen philosophischen Anarchismus, der aus einer strikten Anwendung des entsprechenden Legitimitätskriteriums zu folgen scheint. Und eben diese Kombination, so meine im Folgenden zu erläuternde These, findet sich auch in Rousseaus Politischer Theorie: Rousseau ist ein aposteriorischer bzw. sekundärer Anarchist, da die anspruchsvollen Kriterien für legitime politische Autorität, die er im Gesellschaftsvertrag formuliert, seines Erachtens in den existierenden politischen Gemeinwesen nicht erfüllt werden, so dass keine genuine politische Verpflichtung vorliegt; und er ist ein philosophischer Anarchist, da er daraus nicht den Imperativ ableitet, sich nun auch praktisch-politisch am Umsturz der etablierten illegitimen Ordnung zu beteiligen. Im Unterschied zum Anarchismus finden sich bei ihm darüber hinaus jedoch wichtige Hinweise darauf, welche demokratietheoretischen Implikationen die Konstitution eines legitimen Gemeinwesens für dessen Struktur hat.

\section{Rousseau über die Begründung politischer Verpflichtung}

Die Frage nach dem Verhältnis von Rousseaus Theorie der politischen Verpflichtung zu den eben skizzierten gegenwärtigen Debatten ist bisher kaum thematisiert worden. ${ }^{5}$ Bevor ich zu der auf den ersten Blick überraschenden These komme, dass Rousseau eine Variante des sekundären philosophischen Anarchismus ver-

5 Vgl. jedoch Wolff (1998, S. 48 ff.), der Rousseaus allgemeinen Willen dann aber mit dem Willen der Mehrheit kurzschließt, sowie Stilz (2009), die sich neben Kant vor allem auf Rousseau bezieht, um zu begründen, warum wir einem demokratischen Staat, der unsere Freiheitsrechte realisiert und schützt, gegenüber zum Gesetzesgehorsam verpflichtet sind, dann aber das unten im vierten Abschnitt behandelte Problem der Auflösung politischer Verpflichtung und der zu entsprechenden Urteilen befugten Instanz weitgehend ausblendet. 
tritt, sei daher kurz an die grundlegende Argumentationsstruktur von Rousseaus Theorie erinnert.

Einen guten Ausgangspunkt stellt Rousseaus eigene Charakterisierung im sechsten seiner Briefe vom Berge dar:

„Worin besteht die Einheit des Staates? In der Vereinigung seiner Mitglieder. Und woraus entsteht die Vereinigung seiner Mitglieder? Aus der Verpflichtung [obligation], welche sie alle miteinander verknüpft. Bis hierher ist alles einig. Allein, welches ist die Grundlage dieser Verpflichtung? Hier teilen sich nun die Autoren. Nach einigen ist es die Gewalt, nach anderen die väterliche Autorität, nach wieder anderen der Wille Gottes. Jeder sucht seinen Grundsatz zu behaupten und den des anderen anzugreifen; ich selbst habe es nicht besser gemacht, und indem ich die vernünftigste Partei von denen, die über diese Sache geschrieben haben, befolgte, habe ich die Übereinkunft [convention] der Mitglieder als die Grundlage des politischen Körpers angegeben und die Grundsätze, die den meinigen entgegenliefen, widerlegt. [...] Welche sicherere Grundlage kann die Verpflichtung haben als den freien Willen dessen, der sich verpflichtet [le libre engagement de celui qui s'oblige]? Man kann über jeden anderen Grundsatz streiten, nur nicht über diesen“ (Rousseau 1978, S. 145-146 [OC III, S. 806-807] [Übersetzung modifiziert].

In dieser Zusammenfassung der Grundgedanken des Gesellschaftsvertrags, die sich allerdings etwas voluntaristischer liest als in der ursprünglichen Formulierung, präsentiert Rousseau seine Theorie explizit als Antwort auf das Problem der politischen Verpflichtung. ${ }^{6}$ Rousseau verdeutlicht hier, was er im ersten Buch des Gesellschaftsvertrags zu zeigen versucht hat: Die politische Verpflichtung stellt eine besondere Art der Verpflichtung dar, die weder durch Bezug auf das Modell väterlicher oder göttlicher Autorität, noch auf natürliche Tatsachen wie de factoMacht (ein vermeintliches Recht des Stärkeren) oder intellektuelle Überlegenheit (Expertenwissen), noch, wie Rousseau im Genfer Manuskript argumentiert, in einem vorgängigen natürlichen Recht begründet werden kann. ${ }^{7}$ Im Unterschied zu gängigen moralischen Pflichten handelt es sich bei der politischen Verpflichtung nicht um eine natürliche Pflicht - sie ist vielmehr in der Konvention, der Übereinkunft, der Zustimmung verankert, die Rousseau zufolge den politischen Körper allererst konstituiert. Verpflichtet-sein wird damit - und das ist Rousseaus revolutionäre Neuerung - zurückgeführt auf einen Akt der Selbstverpflichtung, die passive Verpflichtung auf die aktive Verpflichtung (vgl. Bernardi 2006, Kap. 1; Rehm 2012).

Rousseaus Antwort auf das Problem der politischen Verpflichtung kann mithin als immanent bezeichnet werden: Sie ist allein in der spezifischen Art der Konstitution des politischen Körpers begründet - im „Akt [...], durch den ein Volk zum Volk wird“ -, dessen spezifischer Charakter sich mit Bezug auf die Unterschei-

6 Bernardi (2006) rekonstruiert diese Argumentationsrichtung systematisch und begriffsgeschichtlich; auch Stilz (2009, S. 60) betont, die zentrale Frage Rousseaus sei, „What form must political authority take in order to place citizens under a genuine obligation to obey? “.

7 Vgl. Rousseau 2013, S. 174 (OC III, S. 282): „[W]as die Identität der Natur angeht, so ist ihre Wirkung in dieser Hinsicht gleich Null, weil sie für die Menschen ebenso ein Gegenstand des Streits wie der Einigkeit ist und unter ihnen ebenso oft zu Konkurrenz und Neid wie zu gutem Einvernehmen und Einigkeit führt.“ 
dung zwischen einer bloßen „Anhäufung [agrégation]“ und einer „Vereinigung [association] “ erläutern lässt (Rousseau 2013, I.5, S. 23-24 [OC III, S. 359]). Bei ersterer „fehlen das öffentliche Wohl und der politische Körper“, sie ist nicht sui generis, sondern einfach die Summe ihrer Teile. ${ }^{8}$ Der Begriff der politischen Gemeinschaft selbst ist Rousseau zufolge also die Grundlage, auf der die Konzeption der politischen Verpflichtung ausgearbeitet wird, die er vertritt. Daraus folgt auch, dass die Zustimmung zwar eine notwendige Bedingung für das Vorliegen politischer Verpflichtung ist, an sich aber nicht als hinreichend gelten kann, da zum Beispiel ein freiwillig geschlossener Vertrag, mit dem man sich in die Sklaverei verkauft, ebenso ungültig ist wie der Hobbes'sche Vertrag, der Rousseau zufolge ja nicht nur unklug, also ein schlechter deal ist, sondern eine Art Widerspruch in sich: „Schließlich ist eine Vereinbarung nichtig und widersprüchlich, die einerseits unumschränkte Autorität und andererseits grenzenlosen Gehorsam fordert." (Rousseau 2013, I.4, S. 20 [OC III, S. 356]) Zu derselben Schlussfolgerung muss Rousseaus Analyse und Kritik des Betrugsvertrags aus dem Zweiten Diskurs führen: Die Armen stimmen dem Vertrag zwar zu, aber der Gehalt des Vertrags steht seiner Gültigkeit ebenso entgegen wie seine Form und die Bedingungen, unter denen er abgeschlossen worden ist. ${ }^{9}$

Was folgt nun Rousseau zufolge aus der so verstandenen Konstitution der politischen Gemeinschaft für deren Struktur? Die von ihm beschriebene Konstitution des politischen Körpers ist zugleich die Einsetzung des Volkes als Souverän in einer politischen Gemeinschaft der Freien und Gleichen (vgl. Cohen 2010) - ein Bruch mit dem bis dahin gängigen Verständnis von Souveränität als oberster Befehlsgewalt einer (individuellen oder kollektiven) Herrschaftsinstanz über ihre Untergebenen. Wie Judith Shklar (1985, S. 168) schreibt, bewirkt diese Demokratisierung des Souveränitätsbegriffs dessen radikale Transformation: "The sovereign people implies the destruction of sovereignty as a relation between rulers and ruled." Die in der so etablierten egalitär-demokratischen Gemeinschaft konstituierte Form der politischen Freiheit als Autonomie hat zwei Dimensionen: "Being free of constraints imposed by the wills of other individuals and being subject only to self-given laws are merely two different ways of satisfying the ba-

8 Vgl. hierzu auch Rousseaus Kritik im Genfer Manuskript (Rousseau 2013, S. 176 [OC III, S. 284]) an Diderots Bezug auf die „allgemeine Gesellschaft“ der Menschheit, die Rousseau zufolge gerade keine Entität sui generis ist: „Wenn die allgemeine Gesellschaft irgendwo anders existieren würde als in den Systemen der Philosophen, wäre sie, wie ich bereits ausgeführt habe, ein moralisches Wesen mit besonderen Eigenschaften, die sich von den Eigenschaften der partikularen Wesen, die sie konstituieren, unterscheiden.“

9 Wie die substanziellen Bedingungen genau zu verstehen sind, wo also die Grenzen von Rousseaus Voluntarismus verlaufen, ist notorisch schwierig zu entscheiden. Allerdings gilt: Allein der Gehalt, also die Ausrichtung am Gemeinwohl, oder die Form der Reziprozität kann keinerlei Verpflichtung generieren, so dass der eigentliche Grund der Verpflichtung doch in der Zustimmung zu suchen ist. Vgl. etwa Derathé 1995, S. 238-239: „Le citoyen a le devoir d'obéir ou de se soumettre à la volonté générale, non pas parce qu'elle représente l'intéret commun ou un intéret supérieur, mais parce qu'il s'y est formellement engagé par le pacte social. [...] L'obligation d'obéir à la volonté générale a donc son fondement dans le pacte social, dans l'engagement ou le consentement de tous. "In Rousseaus Werk finden sich zudem sowohl Formulierungen, die ein Verständnis des Gesellschaftsvertrags im Sinne tatsächlicher Zustimmung nahelegen, als auch solche, die eher für eine Interpretation als hypothetischer Vertrag sprechen. 
sic condition of freedom, which stipulates that one obey no will other than one's own” (Neuhouser 1993, S. 393).

Wie lässt sich Rousseaus Position vor diesem Hintergrund im Feld der im letzten Abschnitt skizzierten gegenwärtigen Diskussion situieren? Ebenso wie die neueren Konsens- bzw. Zustimmungstheorien der politischen Verpflichtung und der philosophische Anarchismus à la Simmons scheint Rousseau der Ansicht zu sein, dass die Zustimmung der Rechtsadressaten eine notwendige (wenn auch, wie eben gesehen, nicht hinreichende) Bedingung für die Existenz politischer Autorität und damit politischer Verpflichtung darstellt. Die Frage ist dann freilich, wie das Kriterium der Zustimmung von Rousseau genauer verstanden bzw. praktisch operationalisiert wird, wenn es nicht auf den ursprünglichen Gesellschaftsvertrag beschränkt werden soll.

Dieses Problem lässt sich anhand eines Kommentars von T.H. Green (1986, S. 63) etwas präziser fassen, der in seinen berühmten Lectures on the Principles of Political Obligation nach der Zusammenfassung des vertragstheoretischen Grundgedankens wie folgt fortfährt: "So far all is consistent. [...] But after the first generation of those who were parties to the supposed original compact, what is to settle whether anyone is a party to it or not?" Rousseau scheint auf diese Frage eine Antwort zu haben (Rousseau 2013, IV.2, S. 127 [OC III, S. 440]). Zunächst wiederholt er die allgemeine These: „,[D]ie bürgerliche Vereinigung ist der freiwilligste Vorgang auf Erden. Da jeder Mensch frei und als Herr seiner selbst geboren wird, darf ihn niemand, unter welchem Vorwand auch immer, ohne seine Zustimmung zum Untertan machen.“ Dann fügt er hinzu: „Wenn der Staat gegründet ist, bewirkt der Wohnsitz innerhalb seiner Grenzen die Zustimmung; ein Staatsgebiet bewohnen heißt, sich der Souveränität zu unterwerfen.“ Green (1986, S. 63) ist nicht überzeugt:

"This answer, however, will scarcely stand examination. Rousseau himself does not consider that residence in the same region with the original parties to the pact renders those so resident also parties to it. Why should it do so, when the pact has descended to a later generation? It may be argued of course that everyone residing in a settled society, which secures him in his rights of person and property, has the benefit of the society from the mere fact of his residence in it, and is therefore morally bound to accept its laws. But this is to abandon the doctrine of obligation being founded on consent. Residence in a territory governed by a certain sovereign can only be taken to imply consent to the rule of that sovereign, if there is any real possibility of relinquishing it, and this there can scarcely ever be."

Sehen wir uns nun die Fußnote zu der oben zitierten Passage aus dem Gesellschaftsvertrag genauer an, in der Rousseau folgende Einschränkung vornimmt:

„Das gilt immer für einen freien Staat, denn andernfalls können Familie, Besitz, Mangel an Asyl, Not oder Gewalt einen Einwohner entgegen seinem Willen im Land festhalten, und dann bedeutet sein bloßer Aufenthalt nicht länger seine Zustimmung zum Vertrag oder zu dessen Verletzung.“

Diese Erläuterungen sind nicht ganz einfach zu interpretieren. Zum einen drücken sie ein recht konventionelles Argument aus, das auch Green im Sinn hat, nämlich 
dass die Exit-Option den Betroffenen auf realistische Weise offenstehen muss; zum anderen kann man den Verweis auf den „freien Staat“ aber auch stärker auslegen (und damit über die klassische Vertragstheorie hinausgehen): Durch Aufenthalt erwirbt man die Verpflichtung nur in einer Gesellschaft, die wohlgeordnet im Sinne des Gesellschaftsvertrags ist, und hierzu gehört, dass die Bürger kollektiv als Souverän über die Gesetzgebungsgewalt verfügen und mithin allein unter selbstgegebenen Gesetzen leben.

Carol Pateman hat diesen Aspekt von Rousseaus Theorie der politischen Verpflichtung am deutlichsten herausgearbeitet und zugleich radikalisiert. Ihres Erachtens lässt sich das Problem der politischen Verpflichtung Rousseau zufolge nur in einer partizipatorischen politischen Gemeinschaft lösen:

“'[A] uthority' is usually seen in terms of the relationship between citizens and the liberal state. Theorists rarely consider another form of authority relationship; one in which citizens freely create relationships of authority for themselves. Thus definitions of 'authority' usually cannot encompass a democratic form of political organization in which members of a political association, in their capacity as citizens, exercise political authority over themselves as individuals. Or, to make this point slightly differently, authority is usually regarded as one way in which individuals can exercise power over others; it is not seen as a way of giving citizens collective power to, or the ability to, act for themselves. It is seen as a form of control rather than as a means of citizens enlarging their collective capacity to create and take responsibility for their own political lives" (Pateman 1985, S. 136).

Wie bereits gesehen, geht damit ein spezifisches Verständnis von Souveränität einher, das diese nicht mehr als Befehlsgewalt einer (individuellen oder kollektiven) Instanz über alle Subjekte, sondern als kollektives Recht des Volkes zur Gestaltung der eigenen Lebensbedingungen versteht (vgl. zu diesen beiden Souveränitätskonzeptionen auch Kalyvas 2005). Pateman spricht im Fall Rousseaus daher von einem demokratischen bzw. partizipatorischen Gesellschaftsvertrag, der sich in wesentlichen Hinsichten von den Vertragsmodellen der liberalen Tradition unterscheidet, da er eine horizontale statt einer vertikalen Beziehung zwischen den Bürgern etabliert, die den Gesetzesgehorsam nicht einer übergeordneten staatlichen Instanz, sondern sich selbst wechselseitig als Ko-Autoren der Gesetze schulden. Insofern könnte man auch sagen, dass es in Rousseaus idealem Gemeinwesen (wie in Marx' kommunistischer Assoziation) keinen Staat im Sinne eines der Gesellschaft gegenüberstehenden und von ihr vermeintlich unabhängigen Apparats mehr gibt (vgl. auch Levine 1993).

Die Antwort auf Greens Deutung des Problems findet sich mithin darin, dass der Gesellschaftsvertrag Rousseau zufolge nicht einfach als einmaliger Akt zu verstehen ist, zu dem im weiteren die implizite Zustimmung relativ umstandslos unterstellt werden kann; vielmehr muss die demokratische und assoziative Logik des Gesellschaftsvertrags in der institutionellen Struktur und der bürgerlichen Praxis der politischen Gemeinschaft auf Dauer gestellt werden: "It is kept in being, and regularly renewed, through the decisions of the citizens in the participatory political community" (Pateman 1985, S. 152). Auch wenn es vermutlich etwas zu weit gehen würde, die Rousseau'sche Republik in Umwandlung eines Zitats von 
Ernest Renan als „contrat social de tous les jours“ zu bezeichnen, muss der demokratische Gesellschaftsvertrags sich doch in der partizipatorischen Struktur der Rousseau'schen Republik spiegeln, wenn seine verpflichtende Kraft nicht erlöschen soll. Nur in einer demokratisch konstituierten und strukturierten Republik, nur in einem „freien Staat", haben die Bürger demnach eine genuine politische Verpflichtung (auch wenn mit diesem Übergang der Rahmen der reinen Zustimmungstheorie verlassen wird, denn individuelle Zustimmung ist nicht mit der Partizipation unter Gleichen zu identifizieren, obwohl sie mit dieser in einem engen Zusammenhang steht). Wie der oben skizzierte aposteriorische bzw. sekundäre Anarchismus ist Rousseau der Auffassung, dass kein moderner Staat die Kriterien für genuine politische Verpflichtung erfüllt - wie der philosophische Anarchismus folgert er daraus allerdings nicht, dass Ungehorsam in einer nicht den Prinzipien des Gesellschaftsvertrags entsprechenden politischen Ordnung geboten oder auch nur (aus diesem Grund bereits) zulässig wäre. ${ }^{10}$ Insofern es auch in einer solchen Ordnung gute und sogar vorrangige Gründe geben kann, sich an die Gesetze zu halten, sind diese jedoch qualitativ anderer Art und nicht auf die besondere Form politischer Autorität zurückzuführen, die nur in einer wohlgeordneten Gemeinschaft möglich ist.

\section{Die Auflösung politischer Verpflichtung}

Politische Verpflichtungen - also eine Pflicht zum Rechtsgehorsam seitens der Bürger - gibt es für Rousseau, wie gesehen, nur unter genau definierten und sehr anspruchsvollen Bedingungen. Demnach gibt es - entgegen dem ersten Anschein - in den allermeisten Situationen keine politische Verpflichtung. In einem ersten Schritt lassen sich folgende vier Fälle unterscheiden, in denen eine solche Verpflichtung nicht oder nicht mehr gegeben ist. Dabei ist der erste Fall der gängigste:

(1) Wie gesehen, setzt genuine politische Verpflichtung die Existenz eines Gesellschaftsvertrags des richtigen (also dem Modell des Gesellschaftsvertrags entsprechenden) Typs voraus: In allen anderen Fällen ist die Vorstellung oder das Gefühl der Verpflichtetheit bloßer Schein bzw. bloße Ideologie und Rousseaus Position läuft auf die des philosophischen Anarchismus hinaus. Aus dieser Diagnose ergibt sich auch ein praktisches Interesse, das die Vertreter des philosophischen Anarchismus mit Rousseau teilen. Die im Zweiten Diskurs vorgelegte Analyse und Kritik

10 Daher steht es auch nicht im Widerspruch zu der obigen Deutung, wenn Rousseau (1998, S. 330 [OC IV, S. 629]) den Vikar am Ende seines Glaubensbekenntnisses im Emile sagen lässt (also nicht selbst sagt): „Halten wir die öffentliche Ordnung aufrecht, bis uns größere Einsichten zuteil werden. Achten wir die Gesetze eines jeden Landes und stören wir nicht den Kult, den sie vorschreiben; verleiten wir die Bürger nicht zum Ungehorsam, denn wir wissen nicht sicher, ob es zu ihrem Besten ist, wenn sie ihre Meinung gegen andere austauschen; dagegen wissen wir mit Sicherheit, daß der Ungehorsam gegen die Gesetze ein Übel ist.“ Dass Rousseau die bestehende Ordnung auch im Emile nicht für sakrosankt erklärt oder für stabil hält, zeigt die folgende Passage (Rousseau 1998, S. 192 [OC IV, S. 468]): „Ihr verlaßt euch auf die bestehende Gesellschaftsordnung und bedenkt nicht, daß sie unvermeidlichen Veränderungen unterworfen ist, und daß ihr diejenigen, die eure Kinder erleben werden, weder voraussehen noch verhindern könnt. Der Große wird klein, der Reiche arm, der Monarch Untertan. [...] Wir nähern uns einer Krise und dem Jahrhundert der Revolutionen.“ Und in einer Anmerkung zu dieser Passage fügt Rousseau hinzu: „Ich halte es für unmöglich, daß die großen Monarchien Europas noch lange bestehen werden." 
des Gesellschaftsvertrags, der keine legitime Einigung, sondern ein Täuschungsmanöver der Reichen darstellt, das aus prozeduralen wie aus substantiellen Gründen keine Verpflichtung generieren kann, legen eine skeptische Einstellung gegenüber der regelmäßigen Berufung auf die Idee der politischen Verpflichtung und ihrer weitgehenden Akzeptanz unter den Rechtssubjekten nahe - zumal in Situationen, in denen der Glaube an politische Verpflichtung und die entsprechenden Praktiken des Gehorsams, der Unterwerfung und der Loyalität im Interesse der Mächtigen sind. Rousseau und Simmons scheinen darin übereinzustimmen, dass es entgegen der herrschenden Meinung kein Gemeinwesen gibt, das den anspruchsvollen Bedingungen für die Existenz politischer Verpflichtung genügt.

Auch in einem wohlgeordneten Gemeinwesen, also einem Gemeinwesen, dessen Konstitution und Struktur den Prinzipien des Gesellschaftsvertrags entspricht und mit Bezug auf das Rousseau dementsprechend keine anarchistische Position vertritt, kann das Vorliegen politischer Verpflichtung jedoch fraglich werden und zwar auf drei unterschiedliche Weisen.

(2) Wie Rousseau betont, hängt die Gültigkeit, ja die Identifikation einzelner Gesetze als Gesetze (neben ihrer formalen Qualifikation als Gesetze durch ihre Allgemeinheit) an zwei Kriterien, von denen das eine prozedural und das andere substantiell ist: Gesetze müssen zum einen Ergebnis der Gesetzgebung (bzw. zumindest der Ratifikation) durch das Volk sowie zum anderen am Gemeinwohl ausgerichtet sein. Andernfalls handelt es sich nicht um genuine Gesetze - das gilt sowohl für das prozedurale Kriterium: „Jedes vom Volk nicht in Person ratifizierte Gesetz ist nichtig; es ist kein Gesetz." (Rousseau 2013, III.15, S. 114 [OC III, S. 430]), als auch für das substantielle Kriterium:

„[W]enn die niedrigsten Interessen die Frechheit besitzen, sich mit dem geheiligten Namen des öffentlichen Wohls zu schmücken, dann wird der allgemeine Wille verstummen [...und] unter dem Namen von Gesetzen [werden] auf unrechtmäßige Weise ungerechte Verordnungen ausgegeben, die nur partikulare Interessen zum Ziel haben“" (Rousseau 2013, IV.1, S. 124 [OC III, S. 438]). ${ }^{11}$

Damit werden der Mehrheit scheinbar klare Grenzen auferlegt: Ein Gesetz kann mit noch so großer Mehrheit verabschiedet werden, wenn es ein Individuum oder eine Minderheit seiner bzw. ihrer Rechte beraubt - und etwa von der politischen Entscheidungsfindung ausschließt -, hat es keine Gültigkeit und ist daher kein Gesetz (vgl. Stilz 2009, S. 94). In einem solchen Fall kann sogar behauptet werden, dass die Existenz der politischen Gemeinschaft selbst und damit die Grundlage politischer Verpflichtung überhaupt in Frage steht. Von der lokalen Auflö-

11 Es ist wichtig, die Redeweise von einem substantiellen Kriterium hier nicht falsch zu verstehen: Es handelt sich nicht um ein Kriterium, das sich unabhängig vom politischen Prozess etwa von Gemeinwohlexperten anwenden ließe. Insofern wendet Rousseau dieses substantielle Kriterium selbst noch einmal prozeduralistisch und versteht es als eines, auf das sich die Bürger im politischen Prozess beziehen und das sich nicht unabhängig von diesem Prozess, gewissermaßen aus der Beobachterperspektive ausbuchstabieren lässt. Vgl. Maus 2011, S. 345: „[D]ie volonté générale kann nur an dem ,Ort' des empirischen demokratischen Gesetzgebungsverfahrens zum Ausdruck gebracht (oder verfehlt) werden - ist aber genau deshalb einer expertokratischen oder totalitären Usurpation nicht zugänglich. " Dieser Monismus führt freilich auch in Probleme, auf die ich im fünften Abschnitt zurückkommen werde. 
sung politischer Verpflichtung in konkreten Einzelfällen führt Rousseau zufolge demnach ein relativ direkter Weg zum Extremfall der Auflösung des politischen Körpers.

(3) Rousseau unterscheidet strikt zwischen dem Volk als Souverän, dem das alleinige Recht der Gesetzgebung zukommt, und der Regierung als ausführender Instanz, die vom Souverän eingesetzt und mit einem klar umrissenen Auftrag ausgestattet wird. Mit dem Recht der Einsetzung der Regierung - deren Mitglieder letztlich einfache Beamte sind - geht Rousseau zufolge auch das Recht der Absetzung tyrannischer, ja mißliebiger Regierungen einher. Für den Fall erfolgreicher Usurpation diagnostiziert Rousseau besonders deutlich, dass die Bürger nicht mehr zum Gehorsam verpflichtet sind:

„Im Augenblick, in dem die Regierung die Souveränität usurpiert, wird somit der gesellschaftliche Pakt gebrochen, und alle einfachen Bürger kehren rechtmäßig in ihre natürliche Freiheit zurück und sind daher zwar zum Gehorsam gezwungen, aber nicht mehr dazu verpflichtet. Derselbe Fall tritt auch ein, wenn die Glieder der Regierung einzeln die Macht usurpieren, die sie nur korporativ ausüben dürfen “ (Rousseau 2013, III.10, S. 106 [OC III, S. 423]).

In jedem Fall sollte das Volk in regelmäßig wiederkehrenden Versammlungen die Regierung kontrollieren und entscheiden, ob sie noch ihrem klar umrissenen Auftrag nachkommt. Wie Rousseau im Kapitel mit dem Titel „Mittel, um der Usurpation der Regierung vorzubeugen “ ausführt, gibt es

„Zwei Anträge, die man niemals weglassen darf und die getrennt zur Abstimmung gelangen müssen. Der erste: Gefällt es dem Souverän, die gegenwärtige Form der Regierung beizubehalten? Der zweite: Gefällt es dem Volk, die Verwaltung in den Händen jener zu belassen, die damit gegenwärtig betraut sind?" (Rousseau 2013, III.18, S. 121-122 [OC III, S. 435-436]). ${ }^{12}$

Auch wenn Rousseau davor warnt, durch allzu voreilige Entscheidungen politischer Instabilität Vorschub zu leisten, und sich für einen umsichtigen Umgang mit diesen Anträgen ausspricht, macht er doch deutlich, dass es sich bei der Stabilitätsorientierung um „eine politische Maxime und keine Rechtsregel“ handelt, da der Souverän der Regierung gegenüber in keinem Verpflichtungsverhältnis steht, sondern die Autorität gegenüber seinen Beamten stets selbst in den Händen hält (Rousseau 2013, III.18, S. 120 [OC III, S. 435]). Und wie gesehen ist auch die Verpflichtung der einzelnen Bürger gegenüber der Regierung von klaren Bedingungen abhängig. ${ }^{13}$

12 Vgl. auch Rousseau 1978, S. 171 (OC III, S. 826): „Die gesetzgebende Gewalt ist in zweierlei Dingen unteilbar, nämlich darin, Gesetze zu geben und sie zu bewahren, das heißt Aufsicht über die ausübende Gewalt zu führen. Es gibt keinen Staat in der Welt, wo der Souverän diese Aufsicht nicht hat."

13 Wenn Rousseau die Bedeutung des durch Erziehung und Sitten, Feste und Zivilreligion produzierten und stabilisierten Legitimitätsglaubens der Bürger für ihre Identifikation mit dem und Bindung an das Gemeinwesen betont, dann darf das nicht mit von oben verordneter politischer Apathie („Ruhe als erste Bürgerpflicht“) verwechselt werden. Wie Rousseau (2013, III.14, S. 114 [OC III, S. 430]) bemerkt, sind die Bürger verloren, wenn sie „mehr in ihre Ruhe als in die Freiheit verliebt sind“. Vgl. auch unten Anm. 17. 
(4) Im vierten Fall, der Auflösung des politischen Körpers, scheinen die letzten beiden Tendenzen - die Verletzung der prozeduralen und/oder substanziellen Legitimitätsbedingungen in der Gesetzgebung sowie die Usurpation der Legislativgewalt durch die Regierung - zu kumulieren. Geschieht dies, so finden sich die Bürger in einer Situation wieder, die dem ersten Szenario strukturell ähnelt und sich von ihm nur in ihrer zeitlichen Rahmung unterscheidet: Der Gesellschaftsvertrag existiert nicht mehr und mit ihm erlöschen auch alle politischen Verpflichtungen.

Die Schwierigkeiten, mit denen Rousseaus Position konfrontiert ist, lassen sich am einfachsten am Fall einzelner Gesetze illustrieren: Wie gesehen, gibt es Rousseau zufolge prozedurale und substantielle Bedingungen der allgemeinen Verbindlichkeit politischer Entscheidungen. Unter Bedingungen, unter denen der allgemeine Wille in den Akten der Legislative nicht zum Ausdruck kommt, können die Beschlüsse der Mehrheit nicht als Gesetze betrachtet werden - es sind nurmehr „ungerechte Verordnungen“, denen gegenüber man keine Gehorsamspflicht hat, auch wenn man weiterhin zum Gehorsam gezwungen oder aus anderen Gründen zu ihm motiviert sein mag. Wie aber läßt sich diese Unterscheidung, die als solche ja klar genug zu sein scheint, anwenden? Rousseau zufolge kann es hier nur Indizien - „die langen Debatten, die abweichenden Äußerungen und der Tumult künden vom Erstarken der partikularen Interessen und somit vom Niedergang des Staates" (Rousseau 2013, IV.2, S. 126 [OC III, 439]) -, aber keine sicheren Grundlagen oder klaren Kriterien für ein zweifelsfreies Urteil geben, obwohl Extremfälle wohl einfach zu identifizieren sein dürften.

Damit stehen wir vor einem für die Vertragstheorie vertrauten Problem, das bereits Hobbes und Locke beschäftigte, nämlich der Frage nach der in einer solchen Konfliktsituation zum Urteilen berechtigten Instanz - im vorliegenden Fall: Wer ist dazu berechtigt, sich auf den allgemeinen Willen zu beziehen und so die bindende Kraft eines per Mehrheitsbeschluss verabschiedeten Gesetzes in Frage zu stellen? Rousseau scheint mit den anderen Vertragstheoretikern der Ansicht zu sein, dass dieses Urteil über die Verbindlichkeit einzelner Gesetze nicht dem einzelnen Individuum überlassen werden kann, weil dies ins rechtshermeneutische Chaos führen und den durch den Gesellschaftsvertrag erreichten Stabilitäts- und Freiheitsgewinn wieder zunichte machen würde (vgl. Charvet 1999). Im Gesellschaftsvertrag tritt dieses Problem auch in anderen Zusammenhängen auf, etwa wenn Rousseau behauptet:

„Man wird nicht bestreiten, daß der einzelne durch den gesellschaftlichen Pakt von seiner Macht, seinem Eigentum und seiner Freiheit nur jenen Teil abtritt, dessen Gebrauch für die Gemeinschaft wichtig ist, aber man muß auch zugeben, daß allein der Souverän über diese Wichtigkeit entscheidet" (Rousseau 2013, II.4, S. 42 [OC III, S. 373])..$^{14}$

14 Die Präzisierung des letzten Nebensatzes trifft wohl auch auf die folgende, an John Stuart Mills harm principle erinnernde Fussnote zu (Rousseau 2013, IV.8, S. 163 [OC III, 467]): „In der Republik', schreibt der M. d'A., ,ist jeder vollkommen frei in dem, was den anderen nicht schadet.' Hier liegt die unveränderliche Grenze; genauer kann man sie nicht ziehen. “ Die Frage ist natürlich, wer genau dazu berechtigt ist, den Verlauf dieser „unveränderlichen“ Grenze festzustellen, wenn nicht der Souverän, und ob man dessen Feststellung dann nochmals problematisieren kann. 
In den oben geschilderten Fällen steht aber gerade in Zweifel, ob der faktische Souverän - also die Mehrheit des versammelten Volkes - tatsächlich so entscheidet, wie es seinem normativen Rollenverständnis angemessen wäre. Einfach zu behaupten, das entsprechende Urteil müsse dem Souverän überlassen werden, hilft in einer solchen Situation nicht weiter, da es ja um die Möglichkeit der Problematisierung dieses Urteils geht, ohne dass sich einzelne Bürger damit eine besondere Urteilskompetenz anmaßen könnten, die den Rousseau'schen Egalitarismus untergraben würde.

Vor diesem Hintergrund stellt sich die Frage, ob Rousseau die Operationalisierung der von ihm aufgestellten Bedingungen nicht zu streng bzw. einseitig und damit im Widerspruch zu den radikaleren demokratietheoretischen Implikationen seines partizipatorischen Gesellschaftsvertrags durchgeführt hat. Pateman (1985, S. 159) hat einen ähnlichen Punkt im Sinn, wenn sie schreibt:

“There is to be no right of political dissent or resistance in Rousseau's political association. Just as he refuses to extend his arguments to women, so he fails to pursue the logic of the participatory social contract to its conclusion: that the right of dissent and disobedience is implied by the practice of self-assumed political obligation.”

Rousseau lässt zwar die Möglichkeit zu, dass die Mehrheitsentscheidung nicht mit dem allgemeinen Willen kompatibel ist, und vertritt insofern keine rein voluntaristische oder prozeduralistische Position, meint aber, die Frage, wer das entsprechende Urteil zu fällen berechtigt ist, wie Hobbes allein mit Verweis auf die souveräne Instanz selbst beantworten zu können. Dabei scheint er von einer problematischen Alternative auszugehen: Entweder „alle Merkmale des allgemeinen Willens" finden sich in der Mehrheit, dann ist man als Vertreter der Minderheitenmeinung zum Gehorsam verpflichtet und muss sogar zugestehen, dass man sich geirrt hat, oder diese Merkmale sind nicht mehr in der Mehrheit, dann „gibt es keine Freiheit mehr, welcher Partei man sich auch zuwenden mag“ (Rousseau 2012, IV.2, S. 126 [OC III, S. 439]). Wie Pateman richtig bemerkt, impliziert das, dass entweder alle vorbehaltlos der Mehrheit zustimmen müssen oder die politische Gemeinschaft bereits zu existieren aufgehört hat. Dies lässt gerade jenen Zwischenbereich aus dem Blickfeld verschwinden, in dem selbst in einer wohlgeordneten Gesellschaft Politik stattfindet. Damit reproduziert Rousseau eine Alternative, die eher dem Hobbes'schen als dem demokratischen Vertragsmodell zu entsprechen scheint und der er Pateman zufolge nur durch eine stärkere Demokratisierung der Gemeinschaft selbst entgehen könnte: "If these two all-or-nothing alternatives, characteristic of liberal rather than democratic theory, are to be avoided, the right of free judgement and choice must be extended to the minority" (Pateman 1985, S. 161). Der demokratischen Logik des Rousseau'schen Gesellschaftsvertrags würde es daher eher entsprechen, das Problem der politischen Verpflichtung nicht als ein für alle Mal lösbares, sondern als permanentes Problem zu verstehen, das in einer demokratischen Gemeinschaft als solches anerkannt und durch demokratische Institutionen und Praktiken bearbeitet werden muss. Erst eine partielle Auflösung der monistischen Willensbildungsstrukturen würde berücksichtigen können, dass Bürger unterschiedliche Auffassungen des 
allgemeinen Willens in unterschiedlichen Fora zu artikulieren in der Lage sind. Pateman zufolge sollten zu den entsprechenden Praktiken der bürgerschaftlichen Partizipation auch solche des zivilen Ungehorsams und des Dissenses gezählt werden; allerdings geht sie nicht weiter darauf ein, wie eine spezifisch rousseauistische Perspektive auf zivilen Ungehorsam aussehen könnte - eine Frage, auf die ich im letzten Abschnitt zurückkommen werde.

Dass Rousseaus Theorie politischer Verpflichtung einer solchen Erweiterung über die von ihm selbst vertretenen Optionen hinaus offensteht, behauptet auch Brooke (2007), der vier mögliche Weisen unterscheidet, in denen ein Rousseau'scher Bürger seinen politischen Dissens artikulieren kann. Ohne seine Interpretation hier im einzelnen darlegen zu können, seien diese vier Möglichkeiten kurz aufgeführt und kommentiert: (1) Die Strategie des Schweigens stellt eine Art inneres Exil dar, in das das Individuum aus Gewissensgründen geht, ohne aber offen in Konflikt zu den Entscheidungen der Mehrheit zu treten. Wenn es stimmt, dass die Bürger in einem wohlgeordneten Gemeinwesen „gleichsam auf Flügeln zu den Versammlungen“ eilen (Rousseau 2013, III.15, S. 114 [OC III, S. 429]), dann kann man im Umkehrschluss annehmen, dass der Rückzug aus der Versammlung nicht unbedingt privatistisch motiviert oder Ausdruck einer „Was geht mich das an?"“-Einstellung sein muss, sondern selbst ein politischer Akt sein kann, den Rousseau in seiner Theorie zwar nicht als solchen diskutiert, in der Praxis aber doch anerkannt hat. (2) Die Strategie des Exils ist radikaler, kann aber ebenso in grundsätzlichen Erwägungen und einem wohlverstandenen Patriotismus begründet sein wie die erste Strategie. Auch wenn Rousseau selbst natürlich die meiste Zeit seines Lebens Exilant war und etwa die Briefe vom Berge explizit aus der Position des Exilanten verfasst hat, wird auch diese Strategie in seinen Schriften nicht systematisch berücksichtigt. (3) Die Strategie der List zielt auf die Reform des politischen Gemeinwesens von innen durch kluges politisches Handeln, wird von Rousseau jedoch aufgrund seines grundsätzlichen Verdachts gegen strategisches Handeln ebenfalls nicht diskutiert, auch wenn die Rolle des Gesetzgebers (législateur), die Rousseau im Gesellschaftsvertrag (II.7) theoretisch begründet und in seinen Schriften zu Korsika und Polen praktisch einnimmt, vielleicht in diesem Sinne interpretiert werden könnte. (4) Schließlich verweist auch Brooke auf die Praxis des zivilen Ungehorsams als mögliche Artikulationsform politischen Dissenses, die - anders als Rousseau zu meinen scheint, für den Gehorsam und Selbstkritik dem vollständigen Legitimitätsverlust gegenüberstehen - nicht notwendigerweise Ausdruck eines partikularen Willens zu sein hat. Brooke verschenkt die damit theoretisch eröffnete Gelegenheit jedoch gleich wieder, indem er die liberale Theorie des zivilen Ungehorsams von John Rawls als aussichtsreichsten Kandidaten ins Auge fasst, um Rousseaus Republikanismus an dieser Stelle zu ergänzen. Im folgenden letzten Abschnitt werde ich eine andere und etwas spekulative Antwort auf die Frage skizzieren, ob es eine spezifisch rousseauistische Perspektive auf zivilen Ungehorsam als politische Praxis aktiver Bürgerinnen und Bürger geben kann, die sich von Rousseaus Text ein Stück entfernen muss, sich dabei mit Pateman aber auf die demokratietheoretischen Implikationen von Rousseaus partizipatorischem Gesellschaftsvertrag beziehen kann, die radikaler sind, als er selbst zu akzeptieren bereit scheint. 


\section{Eine rousseauistische Perspektive auf zivilen Ungehorsam?}

Um einer Antwort auf die im Titel dieses Abschnitts gestellte Frage näher zu kommen, müssen zunächst zwei Unterscheidungen getroffen werden. Die erste bezieht sich darauf, dass Rousseau, insofern er im Einklang mit dem philosophischen Anarchismus der Ansicht ist, dass es in den allermeisten realexistierenden Staaten keine politische Verpflichtung gibt, Ungehorsam unter ansonsten gleichen Bedingungen nicht für rechtfertigungsbedürftiger als Gehorsam halten kann. Die folgenden Überlegungen beziehen sich primär jedoch nicht auf diesen Fall, sondern auf den Bereich zwischen den beiden Extremen der Verpflichtung zur vorbehaltlosen Zustimmung und der Auflösung der politischen Gemeinschaft, in dem sich auch in der wohlgeordneten Gesellschaft - also in einem im Prinzip legitimen Gemeinwesen - Politik abspielen dürfte.

Zweitens schlage ich eine recht grobe Unterscheidung zwischen drei (einander nicht ausschließenden, sondern ergänzenden) Modellen des zivilen Ungehorsams vor, die mit unterschiedlichen Vorstellungen der Definition, der Rechtfertigung und der Rolle zivilen Ungehorsams verknüpft sind (vgl. Celikates 2010). In einer Minimalbestimmung, auf die sich diese Modelle einigen können müssten, lässt sich ziviler Ungehorsam verstehen als ein (im Unterschied zu legalen Formen des Protests) absichtlich rechtswidriges und (im Unterschied zu ,gewöhnlichen' Straftaten) prinzipienbasiertes Protesthandeln, mit dem (im Unterschied zur in einigen Staaten grundrechtlich geschützten Weigerung aus Gewissensgründen) das politische Ziel verfolgt wird, bestimmte Gesetze, Maßnahmen oder Institutionen zu verändern, zu verhindern bzw. abzuschaffen oder zu ,forcieren' bzw. einzurichten.

Dem ersten Modell zufolge, das wir als romantisch-individualistisch bezeichnen können, lässt sich ziviler Ungehorsam mit Bezug auf ein höheres - entweder natürliches oder göttliches - Gesetz begründen, zu dem das Individuum vermittelt über das eigene Gewissen Zugang hat. Wie wir gesehen haben, scheint Rousseau einem solchen Bezug auf die private Urteilskompetenz des Individuums zumindest im Gesellschaftsvertrag skeptisch gegenüberzustehen, auch wenn sein eigenes Lebensmotto - vitam impendere vero - durchaus im Sinne der normativen Verpflichtung auf das eigene Gewissen verstanden werden könnte, dem auch in anderen Schriften Rousseaus, vor allem im Emile, als „göttliche Stimme“ eine grundlegende Autorität zuerkannt wird (ohne dass die politischen Konsequenzen dieser Ansicht ausbuchstabiert werden würden). Das zweite Modell lässt sich als liberal-konstitutionalistisch bezeichnen und findet sich paradigmatisch in den Arbeiten von John Rawls und Ronald Dworkin ausgeführt, denen zufolge ziviler Ungehorsam dann (und nur dann) legitim ist, wenn auf nachvollziehbare Weise individuelle Rechte oder basale Gerechtigkeitsgrundsätze herangezogen werden können, die durch eine staatliche Institution oder eine Mehrheitsentscheidung verletzt worden sind. Die Rechtfertigung des zivilen Ungehorsams ist demnach auf substantielle moralische Überlegungen angewiesen, die die Grenzen der Ausübung der Volkssouveränität durch Mehrheitsentscheidung bestimmen. Ziviler Ungehorsam ist in dieser Perspektive als von einzelnen Individuen im Namen ihrer Rechte eingeforderte Begrenzung demokratischer Selbstbestimmung zu verste- 
hen. Auch dieses Modell scheint in einem Spannungsverhältnis zu Rousseaus Politischer Theorie zu stehen.

Während ziviler Ungehorsam aus dieser liberalen Perspektive vor allem als Protestform individueller Grundrechtsträger gegen Regierungen und politische Mehrheiten erscheint, die konstitutionell kodifizierte moralische Prinzipien und Werte verletzen, ist er aus Perspektive des dritten Modells, das sich als republikanischdemokratisch charakterisieren lässt, nicht primär als Beschränkung, sondern als Ausdruck der demokratischen Praxis kollektiver Selbstbestimmung zu verstehen, deren Bedeutung nicht zuletzt darin besteht, ein Gegengewicht zu den Erstarrungstendenzen staatlicher Institutionen zu bilden (vgl. Arendt 1986). Ziviler Ungehorsam zielt - anders als das liberale Modell annimmt - diesem Verständnis zufolge nicht primär auf ein substantiell verstandenes Ideal der gerechten Gesellschaft, sondern auf intensivere und/oder extensivere Formen der demokratischen Selbstbestimmung, die es gegen usurpierende Regierungen und politisch organisierte soziale Partikularinteressen zu erstreiten oder zu verteidigen gilt (vgl. etwa Markovits 2005; Celikates 2010). Aus diesem Verständnis der Rolle zivilen Ungehorsams folgt auch eine andere Perspektive auf seine Rechtfertigung, denn diese wird von substantiellen auf prozedurale Argumentationen umgestellt: Nicht dass das Ergebnis politischer Willensbildungs- und Entscheidungsprozesse bestimmten Normen und Werten widerspricht, sondern dass diese Prozesse selbst durch im Rahmen des politischen Systems kaum vermeidbare strukturelle Demokratiedefizite, etwa in den Dimensionen Partizipation, Repräsentation und Deliberation, aber auch durch den Einfluss von Machtasymmetrien auf die öffentliche Debatte, durch hegemoniale Diskurse und ideologische Selbstverständnisse verzerrt werden (vgl. Young 2001), stellt den Einsatzpunkt der republikanisch-demokratischen Konzeption des zivilen Ungehorsams dar.

Nun lassen sich mit Rousseau sicher auch gewissensbasierte und substantiell moralisch begründete Formen des zivilen Ungehorsam denken, denn natürlich ist es möglich, dass die Mehrheit etwas entschieden hat, das inhaltlich falsch ist (genau deshalb unterscheidet Rousseau ja zwischen dem allgemeinen Willen und dem Willen aller). Wie ein solcher Irrtum identifiziert werden soll, ist, wie gesehen, jedoch nicht ganz klar und aus diesem Grund können wir zumindest annehmen, dass die Begründungslasten für substantielle Argumentationen (wie sie die ersten beiden Modelle erfordern) aus einer rousseauistischen Perspektive höher veranschlagt werden müssen als für das prozeduralistische dritte Modell, in dem es letztlich ja darum geht, die egalitaristisch verstandene (Re-)Artikulation des allgemeinen Willens durch das versammelte Volk zu ermöglichen.

Dieses dritte Modell lässt sich über diese naheliegende Verbindung zu einer von Pateman inspirierten radikaldemokratischen Interpretation des Gesellschaftsvertrags hinaus in zumindest zwei Hinsichten als rousseauistisch verstehen, die ich abschließend kurz skizzieren möchte.

(1) Wenn wir Rousseaus politische Soziologie als wesentlichen Teil seines Werkes begreifen, müssen wir seine Annahme ernstnehmen, daß es auch im wohlgeordneten demokratischen Gemeinwesen unweigerlich Tendenzen der Ablösung der Regierung vom Souverän und der Auflösung des politischen Körpers geben wird - und zwar aufgrund sowohl einer institutionellen als auch einer psychologi- 
schen Dynamik, die Rousseau aufgrund seiner realistischen Einschätzung des kaum vermeidlichen Missbrauchs politischer Institutionen sowie einer Art Vorwegnahme von Robert Michels’ „eisernem Gesetz der Oligarchie“ für unvermeidbar hält. Shklar (1998, S. 272-273) nennt diese Dynamik tragisch, denn die Institution der Regierung und ihre strikte Unterscheidung vom Souverän ist ebenso notwendig wie der von ihr ausgelöste Verfall unvermeidlich ist: „Wenn selbst Sparta und Rom untergingen, welcher Staat dürfte da hoffen, ewig zu dauern?"“ (Rousseau 2013, III.11, S. 107 [OC III, S. 424]; vgl. auch Guénard 2005 sowie Rousseau 2013, III.10, S. 104 [OC III, S. 421]: „Das ist das inhärente und unvermeidliche Laster, das seit dem Entstehen des politischen Körpers ohne Unterlaß denselben zu zerstören tendiert, ebenso wie Alter und Tod den Körper des Menschen zerstören.“) In Reaktion auf diese tragischen Notwendigkeiten steht den politischen Akteuren allein eine „politics of prevention“ (Shklar 1985, S. 165 ff.) offen, die den Verfallsprozess zu verlangsamen und einzudämmen, aber nicht dauerhaft zu stoppen vermag. Warum sollte man vor diesem Hintergrund den zivilen Ungehorsam in seiner republikanisch-demokratischen Variante nicht als ein weiteres „Mittel, um der Usurpation der Regierung vorzubeugen“ verstehen? Sicher, Rousseau legt großen Wert auf die Rechtmäßigkeit der von ihm identifizierten Mittel und betont die Gefahr von Spaltungen, institutioneller Instabilität und der Proliferation von partikularen Willen, ${ }^{15}$ aber im Unterschied zum romantischindividualistischen und zum liberal-konstitutionalistischen Modell besteht beim republikanisch-demokratischen Modell des zivilen Ungehorsams, wie gesehen, nicht gleichermaßen die Gefahr, dass eine Gruppe von Bürgerinnen und Bürgern - wenn auch mit guter Absicht - den anderen ihren eigenen partikularen Willen aufzwingt, denn schließlich geht es um die prozeduralen Möglichkeitsbedingungen demokratischer Selbstbestimmung und nicht um die Durchsetzung oder Verhinderung bestimmter Vorhaben oder Werte. ${ }^{16}$ Dass sich auch bei Rousseau ähnliche Überlegungen finden, zeigen folgende Stellen aus den Briefen vom Berge:

„Beinahe alle ihre Bravourleistungen [die Bemühungen der Genfer Obrigkeit, die legislative Macht an sich zu reißen] waren vergeblich, weil sie Widerstand fanden, und weil in einem Staat wie dem eurigen der öffentliche Widerstand [résistance publique] immer dann sicher ist, wenn er sich auf die Gesetze gründet. [...] In einem Staat [...],

15 Allerdings ist Rousseaus diesbezügliche Position etwas komplexer; vgl. Rousseau 2013, II.3, S. 41 (OC III, S. 372): „Wenn es jedoch Teilgesellschaften gibt, muß ihre Anzahl vermehrt und ihrer Ungleichheit vorgebeugt werden, wie Solon, Numa und Servius es hielten. Diese Vorsichtsmaßnahmen sind die einzig richtigen, damit der allgemeine Wille immer aufgeklärt sei und das Volk vor Irrtum bewahrt bleibt."

16 Die Differenz lässt sich kurz am Beispiel der Proteste gegen „Stuttgart 21“ veranschaulichen: Wenn man zivilen Ungehorsam in einer dem dritten Modell entsprechenden Form praktiziert, tritt man für ein transparenteres, genuin demokratisches Verfahren ein - das Ergebnis eines solchen Verfahrens würde man auch dann als legitim akzeptieren, wenn man inhaltlich durchaus noch Zweifel hätte. Radikale Gegner würden das Ergebnis vielleicht auch dann noch ablehnen, wenn es am Ende eines transparenten und demokratischen Verfahrens stünde (ebenso dürfte es militanten Tierschützern normativ betrachtet relativ gleichgültig sein, ob von ihnen als tierquälerisch und daher illegitim erachtete Praktiken der Mehrheit aufoktroyiert oder von ihr in einem demokratischen Verfahren unterstützt werden) - dass sie damit Recht haben könnten, soll freilich nicht ausgeschlossen werden, aber aus einer von Rousseau inspirierten Perspektive scheint offensichtlich, dass ihr Ungehorsam größere Begründungslasten tragen muss. 
wo die Souveränität in den Händen des Volkes liegt, ist der Gesetzgeber immer vorhanden, obgleich er sich nicht immer zeigt. [... Seine Glieder] können immer über die Verwaltung der Gesetze wachen. Dies ist ein Recht, es ist sogar eine Pflicht, die den Personen obliegt und die ihnen zu keiner Zeit genommen werden kann" (Rousseau 1978, S. 160 und 192 [OC III, S. 816 und 845] [Übersetzung modifiziert]).

(2) Hannah Arendt (1986) grenzt zivilen Ungehorsam ebenso von Lobbyismus im Namen von Partikularinteressen ab wie von einem rein moralischen, gewissensbasierten Appell an das Gewissen der Mehrheit. Als genuin politische und demokratische Praxis ist ziviler Ungehorsam ihr zufolge eine Artikulationsform der Macht des Volkes, in der ein horizontaler, nicht vertikaler Gesellschaftsvertrag zum Ausdruck kommt. Ziviler Ungehorsam ist assoziativ, insofern er „die neueste Form der freiwilligen Vereinigungen “ (Arendt 1986, 154) darstellt, die das horizontale, also demokratische Vertragsmodell exemplifiziert. Sicher, Arendts Betonung von Pluralität, Dissens und Konflikt scheint Rousseaus Homogenitäts- und Einheitsemphase entgegenzustehen, ${ }^{17}$ aber der Hinweis, dass ziviler Ungehorsam als kollektive Praxis der Bürgerinnen und Bürger strukturell dieselbe assoziative Logik aufweist wie der demokratisch-partizipatorische Gesellschaftsvertrag Rousseaus und zudem als Gegenmaßnahme zu Tendenzen der institutionellen Verselbständigung verstanden werden kann, eröffnet eine Perspektive, die an Rousseau anschließt, aber auch für die gegenwärtige Diskussion von Interesse sein sollte (vgl. auch Kalyvas 2008, S. 283 ff.).

Auf der Basis von Rousseaus Theorie wird man dabei jedoch nicht zu einer von allen Spannungen freien Position gelangen. Seine Theorie der politischen Verpflichtung bleibt, wie gesehen, in grundlegenden Hinsichten ambivalent - zwischen beinahe absoluter Gehorsamspflicht in seiner „idealen“ und philosophischem Anarchismus in seiner „nicht-idealen“ Theorie (um eine heute gängige Terminologie zu verwenden). Diese Ambivalenzen können jedoch auch als Manifestationen jener tieferen Spannung, ja Antinomie begriffen werden, die Frederick Neuhouser (2012, S. 349) zufolge nur aufgelöst werden kann, wenn wir das Streben danach aufgeben, die eine Quelle vernünftiger Verpflichtung zu identifizieren, die uns politische Konflikte um die Existenz politischer Verpflichtungen ein für allemal zu vermeiden erlauben würde:

„Wir sollten sie [vernünftige Verpflichtung] stattdessen als etwas betrachten, das aus zwei voneinander unabhängigen Quellen stammt, von denen keiner a priori die Autorität zugestanden werden kann, die andere in Konfliktfällen zu übertrumpfen. [...] Philosophie liefert weder einen Algorithmus noch irgendein anderes Verfahren, um jene Art von Auseinandersetzung darüber, was die Vernunft erfordert, beizulegen, die sich ergibt, wenn beispielsweise ein Individuum seine eigene Gemeinschaft nicht länger als eine ansieht, die vom Geist der Gerechtigkeit [...] belebt wird, und ihre Autorität, Gesetze zu erlassen, die es oder die anderen Mitglieder an sie binden, daher nicht mehr anerkennt.“

17 Es gibt freilich auch Stellen, die sich weniger harmonistisch lesen; vgl. Rousseau 2013, III.9, S. 103 (Anm.) (OC III, S. 420): „Ein wenig Unruhe gibt den Seelen neue Kraft, und was die menschliche Gattung wirklich aufleben läßt, ist weniger der Friede als die Freiheit.“ 
Dass sie diese Auseinandersetzungen anerkennen und öffentlich austragen, statt sie zu leugnen und zu unterdrücken, muss letztlich als Strukturmerkmal demokratisch konstituierter und strukturierter politischer Gemeinschaften begriffen werden, insofern diese dem radikaldemokratischen Erbe von Rousseaus Gesellschaftsvertrag verpflichtet bleiben.

\section{Literatur}

Arendt, Hannah. [1970] 1986. Ziviler Ungehorsam. In dies., Zur Zeit. Politische Essays, 119-160. Hamburg: eva.

Barber, Benjamin. 2012. „Occupy Rousseau“ and Challenge Inequality in America. http:// www.huffingtonpost.com/benjamin-r-barber/occupy-rousseau-and-chall_b_1333812. html. Zugegriffen: 1. August 2012.

Berlin, Isaiah. [1952] 2002. Freedom and Its Betrayal. Six Enemies of Human Liberty. Princeton: Princeton University Press.

Bernardi, Bruno. 2006. Le Principe d'obligation. Une aporie de la modernité politique. Paris: Vrin.

Brooke, Christopher. 2007. Aux limites de la volonté générale. Silence, exil, ruse et désobéissance dans la pensée politique de Rousseau. Les études philosophiques 83:425-444.

Celikates, Robin. 2010. Ziviler Ungehorsam und radikale Demokratie - konstituierende vs. konstituierte Macht? In Die Politik und das Politische, Hrsg. Thomas Bedorf und Kurt Röttgers, 274-300. Frankfurt a. M.: Suhrkamp.

Celikates, Robin. 2011. Loyalität und ziviler Ungehorsam. In Handbuch Angewandte Ethik, Hrsg. Ralf Stoecker, Christian Neuhäuser und Marie-Luise Raters, 351-355. Stuttgart: Metzler.

Charvet, John. 1999. Rousseau, the Problem of Sovereignty and the Limits of Political Obligation. In The Social Contract Theorists, Hrsg. Christopher Morris, 205-218. Lanham: Rowman \& Littlefield.

Cohen, Joshua. 2010. Rousseau. A Free Community of Equals. Oxford: Oxford University Press.

Derathé, Robert. [1950] 1995. Jean-Jacques Rousseau et la science politique de son temps. Paris: Vrin.

Durkheim, Emile. [1918] 2008. Le Contrat social de Rousseau. Paris: Kimé.

Green, Thomas Hill. [1895] 1986. Lectures on the Principles of Political Obligation and Other Writings. Cambridge: Cambridge University Press.

Guénard, Florent. 2005. Puissance législative et puissance exécutive. La marche vers le despotisme. In La religion, la liberté, la justice. Un commentaire des Lettres de la montagne de Jean-Jacques Rousseau, Hrsg. Bruno Bernardi, Florent Guénard und Gabriella Silvestrini, 127-145. Paris: Vrin.

Halbwachs, Maurice. 1943. Introduction, notes et commentaire. In Jean-Jacques Rousseau: $\mathrm{Du}$ Contrat social, Paris: Aubier.

Horton, John. 2010. Political Obligation. Houndmills: Palgrave Macmillan.

Kalyvas, Andreas. 2005. Popular Sovereignty, the Constituent Power, and Democracy. Constellations 12:223-244.

Kalyvas, Andreas. 2008. Democracy and the Politics of the Extraordinary. Cambridge: Cambridge University Press. 
Levine, Andrew. 1993. The General Will. Rousseau, Marx, Communism. Cambridge: Cambridge University Press.

Markovits, Daniel. 2005. Democratic Disobedience. Yale Law Journal 114:1897-1952.

Maus, Ingeborg. 2011. Über Volkssouveränität. Elemente einer Demokratietheorie. Berlin: Suhrkamp.

Neuhouser, Frederick. 1993. Freedom, Dependence, and the General Will. The Philosophical Review 102:363-395.

Neuhouser, Frederick. 2012. Pathologien der Selbstliebe. Freiheit und Anerkennung bei Rousseau. Berlin: Suhrkamp.

Pateman, Carole. 1970. Participation and Democratic Theory. Cambridge: Cambridge University Press.

Pateman, Carole. [1979] 1985. The Problem of Political Obligation: A Critical Analysis of Liberal Theory. Berkeley: University of California Press.

Preuss, Ulrich K. 1984. Politische Verantwortung und Bürgerloyalität. Frankfurt a. M.: Fischer.

Rehm, Michaela. 2012. Obligation in Rousseau. Making Natural Law History? Annual Review of Law and Ethics/Jahrbuch für Recht und Ethik 20 (im Erscheinen).

Rinderle, Peter. 2005. Der Zweifel des Anarchisten. Frankfurt a. M.: Klostermann.

Rousseau, Jean-Jacques. 1964. Euvres complètes, Bd. III. Paris: Gallimard.

Rousseau, Jean-Jacques. [1764] 1978. Briefe vom Berge. In ders.: Schriften, Bd. 2. München: Hanser.

Rousseau, Jean-Jacques. [1762] 1998. Emil oder Über die Erziehung. Paderborn: Schöningh.

Rousseau, Jean-Jacques. [1762] 2013. Vom Gesellschaftsvertrag oder Prinzipien des Staatsrechts. Berlin: Suhrkamp (im Erscheinen).

Shklar, Judith. [1969] 1985. Men and Citizens. A Study of Rousseau's Social Theory. Cambridge: Cambridge University Press.

Shklar, Judith. [1979] 1998. Reading the Social Contract. In dies.: Political Thought and Political Thinkers, 262-275. Chicago: University of Chicago Press.

Simmons, A. John. 2001. Philosophical Anarchism. In ders.: Justification and Legitimacy, 102-121. Cambridge: Cambridge University Press.

Simmons, A. John. 2008. Political Obligation and Authority. In The Blackwell Guide to Social and Political Philosophy, Hrsg. Robert L. Simon, 17-37. Oxford: Blackwell.

Stilz, Anna. 2009. Liberal Loyalty. Freedom, Obligation, and the State. Princeton: Princeton University Press.

Wolff, Robert Paul. [1970] 1998. In Defense of Anarchism. Berkeley: University of California Press.

Young, Iris Marion. 2001. Activist Challenges to Deliberative Democracy. In Political Theory 29: 670-690.

\section{Autorenangaben}

Dr. phil. Robin Celikates, Associate Professor,

University of Amsterdam, Department of Philosophy, Oude Turfmarkt 145,

1012 GC Amsterdam, The Netherlands,

r.celikates@uva.nl 